\title{
Expression and potential prognostic value of histone family gene signature in breast cancer
}

\author{
WENTING XIE $^{1 *}$, JIAJIA ZHANG $^{1 *}$, PENG ZHONG $^{1 *}$, SHANSHAN QIN $^{1}$, HAN ZHANG $^{1}$, \\ XIN FAN ${ }^{1}$, YUZHEN YIN ${ }^{1}$, RUIPENG LIANG $^{2}$, YALI HAN $^{3}$, YINA LIAO $^{3}$, \\ XIAQING YU ${ }^{1}$, HUIDENG LONG ${ }^{1}$, ZHONGWEI LV ${ }^{1}, \mathrm{CHAO} \mathrm{MA}^{1}$ and FEI YU ${ }^{1}$ \\ ${ }^{1}$ Department of Nuclear Medicine, Shanghai Tenth People's Hospital, Tongji University School of Medicine, \\ Shanghai 200072; ${ }^{2}$ Medical College, Anhui University of Science and Technology, Huainan, Anhui 232001; \\ ${ }^{3}$ Shanghai Center of Thyroid Diseases, Shanghai Tenth People's Hospital, Tongji University, Shanghai 200072, P.R. China
}

Received December 28, 2018; Accepted October 11, 2019

DOI: $10.3892 /$ etm.2019.8131

\begin{abstract}
Breast cancer (BC) is the most common type of malignancy among females worldwide. Histone modifications, which are the major post-translational modifications, have a significant role in cancer development and prognosis. However, whether histone family genes may serve as potential prognostic biomarkers for BC patients has remained elusive. In the present study, RNA-sequencing data were obtained from The Cancer Genome Atlas (TCGA). Differentially expressed genes were identified and Gene Ontology and Kyoto Encyclopedia of Genes and Genomes pathway functional enrichment analysis was performed. As histone family genes have been reported to be associated with cervical cancer, the present study hypothesized that histone family genes are associated with gynecological tumors. Histone family genes, including histone cluster $1 \mathrm{H} 1 \mathrm{~A}$ family member B (HIST1H1B), HIST1H2AJ, HIST1H2AM, HIST1H2BI, HIST1H2BO, HIST1H3B, HISTIH3F, HISTIH3H, HISTIH4C and HIST1H4D, were upregulated and identified as hub genes in the protein-protein interaction network. In addition, Oncomine and the Human Protein Atlas were used to further verify the expression levels of histone gene sets. The PrognoScan database was then used to investigate the association between expression and prognostic value regarding cancer patient survival. The present results indicated that higher expression of histone gene sets was associated with poor overall survival, relapse-free
\end{abstract}

Correspondence to: Professor Chao Ma or Professor Fei Yu, Department of Nuclear Medicine, Shanghai Tenth People's Hospital, Tongji University School of Medicine, 301 Yanchang Road, Shanghai 200072, P.R. China

E-mail: ponymachao@163.com

E-mail: yufei021@sina.com

*Contributed equally

Key words: bioinformatics, histone family gene, breast cancer, the Cancer Genome Atlas survival and distant metastasis-free survival of BC patients. The differential expression of histone family genes between $\mathrm{BC}$ and normal samples was validated by reverse transcription-quantitative PCR. Finally, to determine the clinical role of histone family genes in $\mathrm{BC}$, the correlations between histone family genes expression and clinical characteristics were investigated through data collected from TCGA. Therefore, the present study indicates that histone gene sets may be used as prognostic factors for survival prediction for BC patients.

\section{Introduction}

Breast cancer (BC) is the most common type of malignancy among females and represents a serious public health issue. It is a heterogeneous disease that is the leading cause of cancerassociated death among females. For 2018, 2.1 million newly diagnosed cases have been estimated (1). According to cancer statistics, the incidence of BC has increased from 2005 to 2014 , including yearly increases by $0.3-0.4 \%$ per year among Hispanic and black females and $1.7 \%$ per year among Asians/ Pacific islanders (2). Treatments include surgery, radiation and drug therapy. However, the treatment of patients with metastatic BC is challenging $(3,4)$. Numerous biomarkers have been determined for $\mathrm{BC}$ but their application has rarely been implemented in clinical practice (5). Therefore, exploration of novel biomarkers for BC detection, screening, diagnosis, prognostication and treatment monitoring, is urgently required.

Epigenetic modifications are reversible and heritable processes, which are involved in mechanisms associated with the occurrence of cancer without causing any changes in the DNA sequence (6). Furthermore, epigenetic alterations may serve as biomarkers for the detection, prognosis and treatment of cancer (7). Histone modifications are the major type of epigenetic modifications (8). Normally, histone proteins with abundant lysine and arginine residues bind to negatively charged linear DNA to form nucleosomes. The histone family includes histones $\mathrm{H} 1, \mathrm{H} 2 \mathrm{~A}, \mathrm{H} 2 \mathrm{~B}, \mathrm{H} 3$ and $\mathrm{H} 4$. The four core histone proteins, $\mathrm{H} 2 \mathrm{~A}, \mathrm{H} 2 \mathrm{~B}, \mathrm{H} 3$ and $\mathrm{H} 4$, form an octamer. These histones may be modified by a large number of enzymes and are associated with multiple cancers. Histone variant H2A.Z.1 has been reported to have an oncogenic role in hepatocellular 
carcinoma via accelerating the cell cycle transition and epithelial to mesenchymal transition (9). Another previous study suggested that histone variant H2A.Z may be a novel target for BC therapy (10). The transforming growth factor- $\beta$ / protein arginine methyltransferase 5/methylosome protein 50 axis was indicated to regulate transcriptional activation and repression of cancer cell invasion pathways through histone $\mathrm{H} 3$ and $\mathrm{H} 4$ arginine methylation (11). Furthermore, loss of histone H4K20 trimethylation is associated with cell invasion in vitro and may be used as an independent marker to predict poor prognosis in BC patients (12). Although previous large-scale studies suggest that histone genes are involved in numerous types of cancer, a systematic, comprehensive analysis of histone family genes as prognostic markers in BC has not been previously performed.

In the present study, mRNA expression data of breast tumor and normal tissues were downloaded from The Cancer Genome Atlas (TCGA) database and differences in gene expression were assessed. The edgeR package of $\mathrm{R}$ software was used to determine significantly differentially expressed genes (DEGs). The molecular functional and pathway enrichment of these DEGs was assessed using the Database for Annotation, Visualization and Integrated Discovery (DAVID). Next, a closely connected cluster was constructed using the Molecular Complex Detection (MCODE) plug-in of Cytoscape. A previous study reported that histone family genes may serve as prognostic factors for cervical cancer and it can be hypothesized that they are associated with gynecological tumors (13). Thus, they were determined as hub genes in $\mathrm{BC}$ with the criterion of degrees $\geq 10$. To further validate the present results, the Oncomine online platform was used to assess the expression levels of histone family genes. In addition, the association between the expression levels and the prognostic value of histone genes in $\mathrm{BC}$ patients was analyzed. Finally, the differential expression of histone family genes between $\mathrm{BC}$ and normal samples was validated by reverse transcription-quantitative (RT-q)PCR.

\section{Materials and methods}

RNA expression data mining. The RNA sequencing data of 1,208 samples associated with breast carcinoma were obtained from TCGA (https://cancergenome.nih.gov/; accession date, September 14, 2018), and were retrieved using all of the following key words simultaneously: Primary site, breast; program name, TCGA; project ID, TCGA-BRCA; gender, female; work flow type, HTseq-counts; data category, transcriptome profiling; data type, gene expression quantification (14). The mRNA expression data were grouped into 1,096 BC samples and 112 normal breast tissues. These data are publicly accessible and there was no further ethical approval from the Ethics Committee.

Identification of DEGs. The DEGs between normal samples and $\mathrm{BC}$ were selected using the edgeR package in $\mathrm{R}$ (v3.5.1). Edge $R$ is a Bioconductor software package for selecting differences in replicated count data (15). Fold-change (FC) analysis was based on the two groups (tumor tissue and normal tissue). The DEGs were then obtained using an unpaired $t$-test. $\mathrm{P}<0.0001$ and $\log \mathrm{FCl} \geq 4$ were set as cut-off values based on

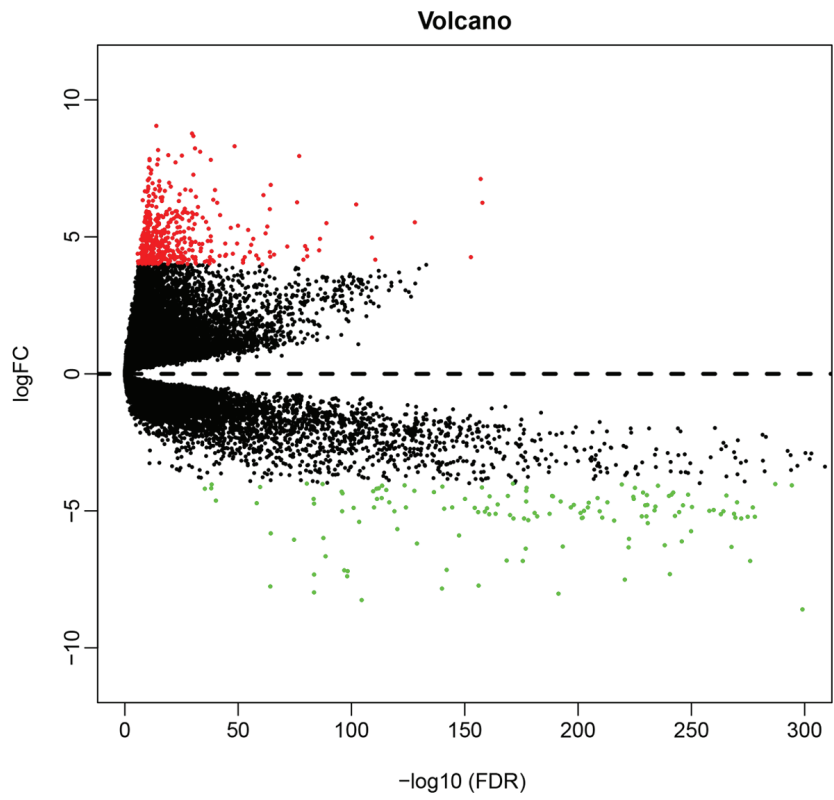

Figure 1. Volcano plots of DEGs in breast carcinoma and normal breast samples. Log FC is displayed on the $y$-axis and the $-\log 10$ (FDR) on the $\mathrm{X}$-axis. FDR refers to the calibrated P-value. Red and green dots represent up-regulated and downregulated genes, respectively. $\mathrm{P}<0.0001$ and $\mid \log \mathrm{FCl}$ $\geq 4$ were used as cut-off criteria. DEGs, differentially expressed genes; FDR, false discovery rate; FC, fold-change.

the Benjamini-Hochberg method. A volcano plot was drawn to represent the DEGs.

Kyoto Encyclopedia of Genes and Genomes (KEGG) and Gene Ontology (GO) enrichment analyses of DEGs. GO functional enrichment analysis of DEGs and KEGG signaling pathway analysis was performed using DAVID (https://david. ncifcrf.gov/; version 6.8). DAVID is an online bioinformatics enrichment tool for comprehensive analysis of the functions of genes $(16,17)$. GO enrichment analysis is an important bioinformatics tool to annotate genes accumulated in the categories 'biological process', 'molecular function' and 'cellular component' $(18,19)$. KEGG is an encyclopedia of genes and genomes, which may be used for pathways enrichment analysis of lists of genes (20). $\mathrm{P}<0.05$ was set as the cut-off criterion.

Protein-protein interaction (PPI) network construction and analysis. The Search Tool for the Retrieval of Interacting Genes (STRING; https://string-db.org/; version 10.5) was applied to construct the PPI network (21). Furthermore, acknowledgement of interactions between proteins may provide further understanding of the complex mechanisms of tumor development. In the present study, the PPI network was built using STRING. A combined score of $>0.4$ was considered to indicate statistical significance. Cytoscape (version 3.6.1), which is a software platform for bioinformatics analysis (22), was used for visualizing PPI.

Hub gene selection and analysis. In the present study, a degree of $\geq 10$ was set as the criterion for selection of hub genes. MCODE (version 1.5.1) is a plugin of Cytoscape which can identify densely connected regions of a given network based on topology. The networks from STRING were visualized 
Table I. GO and Kyoto Encyclopedia of Genes and Genomes pathway enrichment analysis of differentially expressed genes in breast carcinoma samples.

\begin{tabular}{|c|c|c|c|}
\hline Term & Description & Gene count & P-value \\
\hline \multicolumn{4}{|l|}{ Upregulated } \\
\hline GO:0032200 & Telomere organization & 9 & $6.97 \times 10^{-10}$ \\
\hline GO:0044267 & Cellular protein metabolic process & 14 & $1.73 \times 10^{-9}$ \\
\hline GO:0006335 & DNA replication dependent nucleosome assembly & 9 & $3.13 \times 10^{-9}$ \\
\hline GO:0005576 & Extracellular region & 60 & $4.86 \times 10^{-15}$ \\
\hline GO:0000786 & Nucleosome & 16 & $4.47 \times 10^{-13}$ \\
\hline GO:0001533 & Cornified envelope & 10 & $3.60 \times 10^{-9}$ \\
\hline GO:0046982 & Protein heterodimerization activity & 20 & $3.58 \times 10^{-6}$ \\
\hline GO:0005198 & Structural molecule activity & 14 & $9.47 \times 10^{-6}$ \\
\hline GO:0004890 & GABA-A receptor activity & 5 & $6.83 \times 10^{-5}$ \\
\hline hsa05322 & Systemic lupus erythematosus & 16 & $7.53 \times 10^{-11}$ \\
\hline hsa05034 & Alcoholism & 17 & $4.24 \times 10^{-10}$ \\
\hline hsa04080 & Neuroactive ligand-receptor interaction & 14 & $3.72 \times 10^{-5}$ \\
\hline \multicolumn{4}{|l|}{ Downregulated } \\
\hline GO:0008307 & Structural constituent of muscle & 13 & $1.43 \times 10^{-16}$ \\
\hline GO:0003779 & Actin binding & 20 & $2.00 \times 10^{-13}$ \\
\hline GO:0051373 & FATZ binding & 6 & $3.92 \times 10^{-6}$ \\
\hline GO:0030018 & $\mathrm{Z}$ disc & 18 & $2.27 \times 10^{-17}$ \\
\hline GO:0030017 & Sarcomere & 11 & $2.35 \times 10^{-13}$ \\
\hline GO:0031674 & I band & 8 & $2.71 \times 10^{-10}$ \\
\hline GO:0030049 & Muscle filament sliding & 18 & $1.64 \times 10^{-26}$ \\
\hline GO:0006936 & Muscle contraction & 20 & $9.30 \times 10^{-21}$ \\
\hline GO:0060048 & Cardiac muscle contraction & 12 & $4.09 \times 10^{-14}$ \\
\hline hsa04260 & Cardiac muscle contraction & 8 & $3.65 \times 10^{-6}$ \\
\hline hsa04152 & AMPK signaling pathway & 9 & $1.01 \times 10^{-5}$ \\
\hline hsa03320 & PPAR signaling pathway & 7 & $2.42 \times 10^{-5}$ \\
\hline
\end{tabular}

GO, Gene Ontology; GABA-A, $\gamma$-aminobutyric acid-a; PPAR, peroxisome proliferator-activated receptor; AMPK, AMP-activated protein kinase.

using Cytoscape and the subnetworks were drawn by MCODE. The selection criteria were set as follows: MCODE scores, >5; degree cut-off, 2; node score cut-off, 0.2; Max depth, 100; and k-score, 2.

Expression data analysis. The expression data of histone family genes in BC vs. normal tissue were obtained via the Oncomine online database (23). The parameters were set as follows: P-value $<10^{-4}$; FC, $>2$; and gene ranking, top $10 \%$. The immunohistochemistry results on the expression of the histone family proteins in BC were retrieved from the Human Protein Atlas (HPA) database (24).

Survival analysis of hub genes. For survival analysis for hub genes, PrognoScan (http://www.prognoscan.org/) was employed, which is a useful tool for researching the biological association between gene expression and clinical prognosis based on public cancer microarray datasets (dataset numbers provided in Table SV) (25). A Cox proportional hazards model $\mathrm{P}<0.05$ was considered to indicate statistical significance and associated data were displayed in the Kaplan Meier plot.
Ethics statement and clinical specimens. The acquisition of tissue specimens for the present study was approved by the Ethical Committee of Shanghai Tenth People's Hospital (approval no. 107 SHSY-IEC-4.0/19-24/01). Each patient provided written informed consent prior to participating in the study. Fresh BC samples and para-carcinoma tissues were collected from patients who had undergone surgical resection between April and May 2019 in Shanghai Tenth People's Hospital. The authors collected samples from a total of seven patients. There were seven cancer tissues and ten normal tissues, among which three normal tissues were the repetitive tissues belonging to the seven patients (Table SI). A total of seven $\mathrm{BC}$ primary tumor tissues and 10 adjacent non-tumor tissues were collected.

$R N A$ isolation and RT-qPCR. According to the manufacturer's protocols, total RNA was isolated from 10 normal breast tissues and seven BC samples using TRIzol reagent (Invitrogen; Thermo Fisher Scientific, Inc.). A NanoDrop ONE spectrophotometer (Thermo Fisher Scientific, Inc.) was used to measure the total RNA concentration. RNA was used 
A

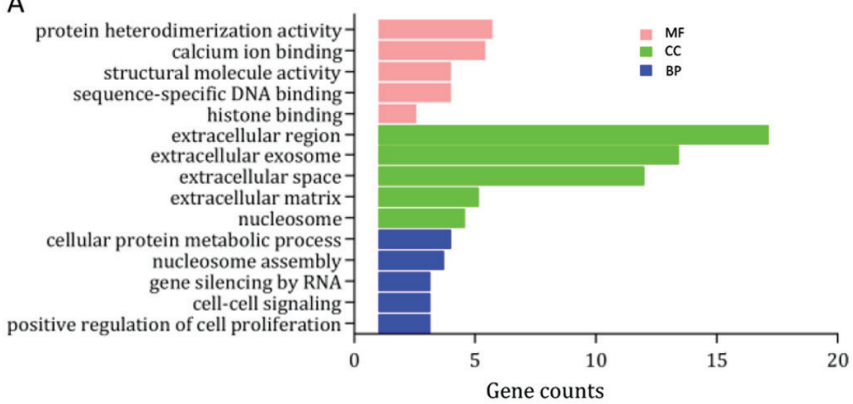

B

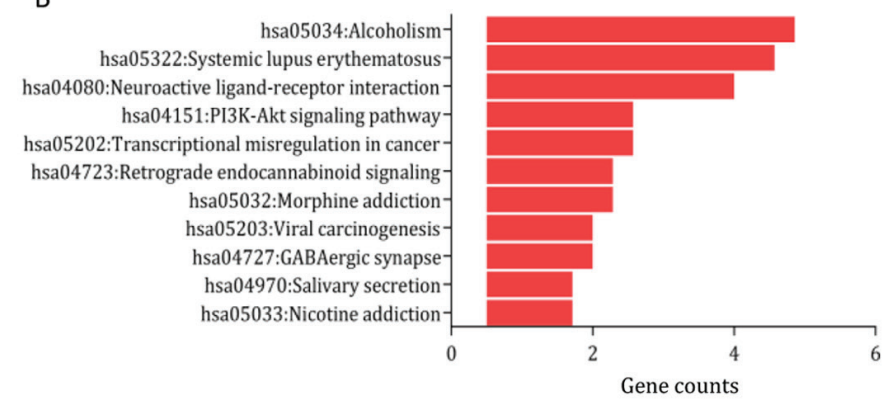

Figure 2. GO and KEGG analysis of upregulated DEGs. (A) GO analysis of upregulated DEGs. Gene counts are displayed on the x-axis and GO function enrichment on the y-axis. (B) KEGG analysis of upregulated DEGs. Gene counts are displayed on the x-axis and KEGG pathway analysis on the y-axis. KEGG, Kyoto Encyclopedia of Genes and Genomes; GO, Gene Ontology; DEG, differentially expressed gene; MF, molecular function; BP, biological process; CC, cellular component; Akt, protein kinase B; PI3K, phosphatidylinositol 3 kinase; GABA, $\gamma$-aminobutyric acid-a.

Table II. Subnetwork module analysis by Molecular Complex Detection plug-in.

\begin{tabular}{lccl}
\hline Score & Nodes & Edges & \multicolumn{1}{c}{ Node IDs } \\
\hline 17.167 & 37 & 309 & MYBPC2, ACTA1, ATP2A1, HIST1H3B, TCAP, HIST1H2AI, \\
& & TTN, NEB, ACTL8, HIST1H4C, HIST1H3J, HIST1H3F, \\
& & HIST1H3H, HIST1H4D, MYL3, TNNC2, HIST1H4B, HIST1H4L, \\
& & HIST1H2BI, TNNC1, HIST1H2AM, HIST1H1B, MYLPF, \\
& & HIST1H2BO, MYL2, HIST1H3I, MYH7, TNNI1, MYL1, \\
& & & HIST1H2AJ, HIST1H2BM, DES, MYH1, TNNI2, TNNT3, \\
& & & MYH2, ACTN2 \\
9.882 & 18 & & OXSS1R, LIPE, FABP4, APOB, GNGT1, INS, CCKBR, TRH, \\
& & & ADIPOQ, GNG13 \\
7 & 7 & & ASB10, ASB15, ASB11, ASB5, FBXO40, UBE2C, KBTBD10 \\
5 & 5 & & GABRA5, GABRQ, GABRA3, GABRA1, GABRG2 \\
4.5 & 5 & CST4, HTN1, CST2, CST5, CST1 \\
4 & 5 & 9 & FBP2, PYGM, GYS2, PCK1, ENO3 \\
4 & 4 & 8 & LCE1F, LCE2C, LOR, LCE1A \\
3.333 & 4 & 6 & NEUROD1, NKX2-2, PDX1, IAPP \\
3.333 & 4 & 5 & ADH1A, ADH1B, HSD17B13, DHRS7C \\
3 & 3 & 5 & LDB3, MYPN, MYOZ1
\end{tabular}

Node Score Cut-off: 0.2; Haircut: true; Fluff: false; K-Core: 2; Max. Depth from Seed: 100.

for first-strand cDNA synthesis in a reaction (final volume, $10 \mu \mathrm{l}$ ) comprising $1 \mu \mathrm{l}$ RNA, according to the protocol of PrimeScript $^{\mathrm{TM}} \mathrm{RT}$ reagent kit (Takara Bio Inc.). The RT conditions were as follows: reverse transcription at $37^{\circ} \mathrm{C}$ for $5 \mathrm{~min}$; inactivation of reverse transcriptase at $85^{\circ} \mathrm{C}$ for $5 \mathrm{sec} ; 4^{\circ} \mathrm{C}$ hold. qPCR was performed using the $\mathrm{C} 1000$ Thermal Cycler (Bio-Rad Laboratories, Inc.) in a reaction (final volume, $25 \mu \mathrm{l}$ ) comprising $2 \mu \mathrm{l} \mathrm{cDNA}$ with the following conditions: Initial denaturation for 1 cycle at $95^{\circ} \mathrm{C}$ for $30 \mathrm{sec} ; 40$ cycles of $95^{\circ} \mathrm{C}$ for $5 \mathrm{sec}$ and $60^{\circ} \mathrm{C}$ for $30 \mathrm{sec}$; PCR primer sequences are listed in Table SII. GAPDH was used as the endogenous control and the $2^{-\Delta \Delta \mathrm{Cq}}$ method was used to analyze the relative expression levels (26).
Statistical analysis. Values are expressed as the mean \pm standard deviation. Student's t-test was used to evaluate the differences between two groups. RNA expression profiling information was used to calculate the Median (M). The Mann-Whitney U test was used to evaluate the differences between two groups in SPSS Statistics version 20.0 software (IBM Corp.). $\mathrm{P}<0.05$ was considered to indicate a statistically significant difference.

\section{Results}

Identification of DEGs in BC. The gene expression data of a total of 1,208 cases, including 1,096 BC samples and 
A

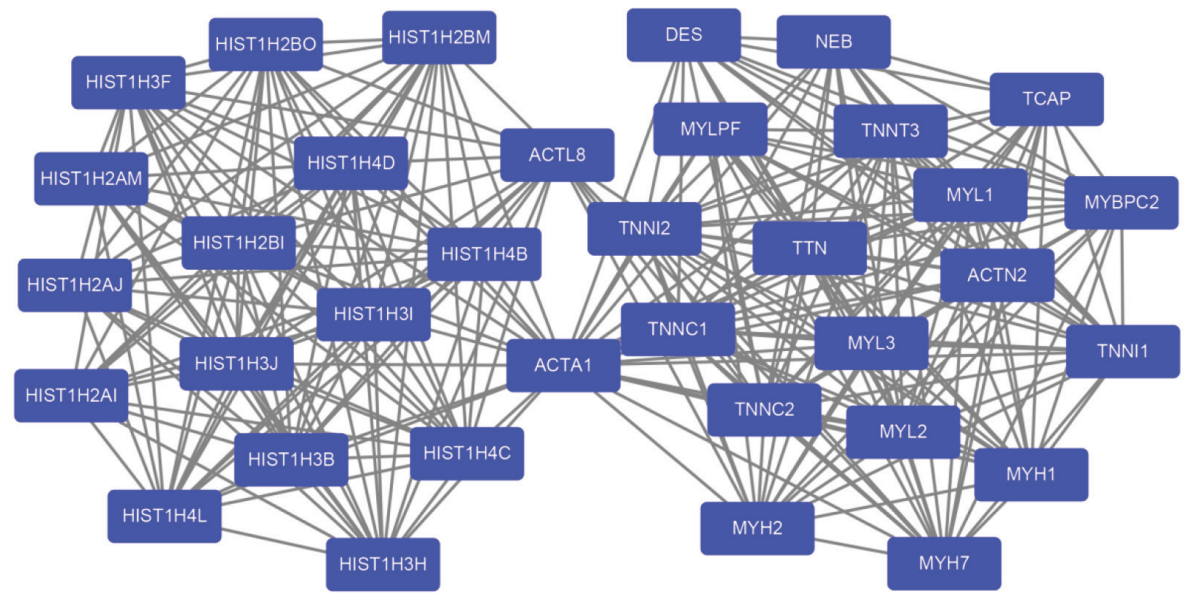

B

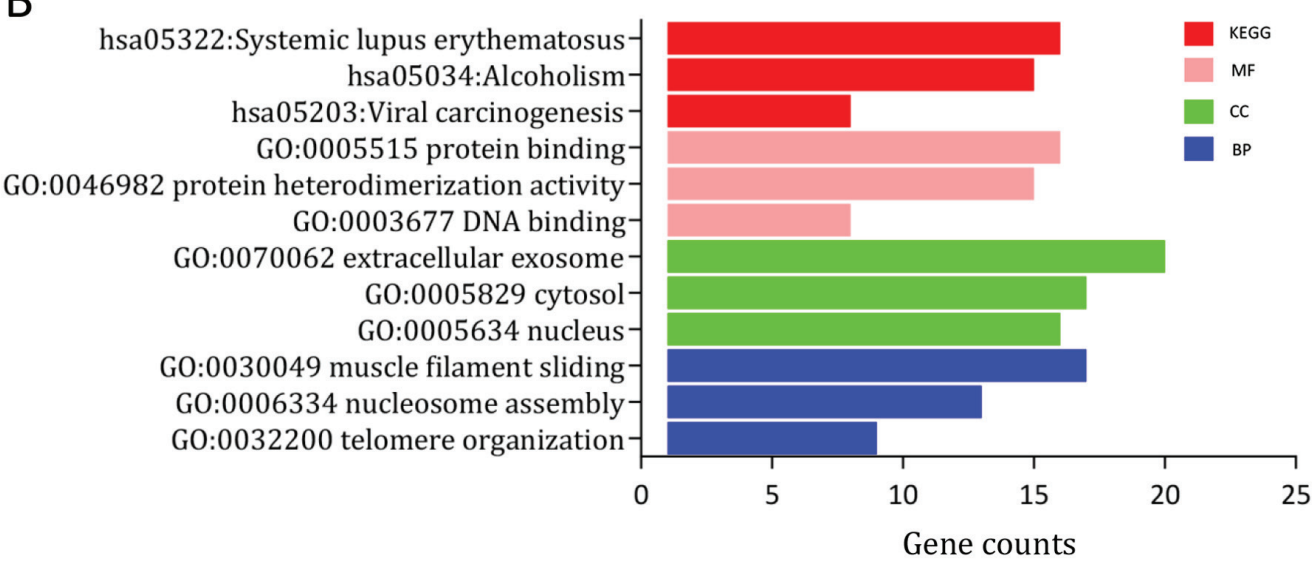

Figure 3. Sub-network construction and analysis. (A) The most significant cluster of DEGs determined using the Molecular Complex Detection plug-in. (B) GO and KEGG enrichment analysis of the most sub-cluster genes by the Database for Annotation, Visualization and Integrated Discovery online platform. KEGG, Kyoto Encyclopedia of Genes and Genomes; GO, Gene Ontology; DEG, differentially expressed gene; MF, molecular function; BP, biological process; CC, cellular component.

112 normal samples in multiple patients, were downloaded from TCGA. $\mathrm{P}<0.0001$ and $\mid \log \mathrm{FCl} \geq 4$ were used as cut-off criteria. Through this analysis, a total of 525 DEGs were determined, of which 366 were upregulated and 155 were downregulated (Fig. 1).

GO and KEGG enrichment analysis of DEGs. DAVID was used to annotate the DEGs, including GO function and KEGG pathway enrichment. The results for the upregulated and downregulated genes are provided in Table I. In the category 'biological process' the upregulated DEGs were enriched in 'DNA replication-dependent nucleosome assembly', 'cellular protein metabolic process' and 'telomere organization' (Fig. 2A), and the downregulated DEGs were enriched in 'muscle filament sliding', 'muscle contraction' and 'cardiac muscle contraction' (Fig. S1A). In the category 'molecular function', the upregulated DEGs were significantly enriched in ' $\gamma$ aminobutyric acid A receptor activity', 'structural molecule activity' and 'protein heterodimerization activity' (Fig. 2A), while the downregulated DEGs were enriched in 'actin binding', 'zinc ion binding', and 'structural constituent of muscle' (Fig. S1A). In addition, in the GO category 'cellular component', the upregulated DEGs were mainly enriched in the terms 'cornified envelope', 'nucleosome' and 'extracellular region' (Fig. 2A), while the downregulated DEGs were significantly enriched in 'I band', 'sarcomere' and 'Z disc' (Fig. S1A). KEGG pathway analysis suggested that the upregulated DEGs were mainly enriched in 'systemic lupus erythematosus (SLE)', 'alcoholism' and 'neuroactive ligandreceptor interaction' (Fig. 2B), while the downregulated DEGs were mainly enriched in the 'peroxisome proliferator activated receptor signaling pathway', 'protein kinase AMP-activated catalytic subunit $\alpha 1$ signaling pathway' and 'cardiac muscle contraction' (Fig. S1B).

PPI network construction and hub gene screening. Next, the PPI network of the DEGs was constructed using STRING with visualization by Cytoscape (Fig. S2). The MCODE plug-in, which is based on topology, was used to identify close clusters in order to locate highly connected regions. The score of cluster modules are presented in Table II. The score of the most significant cluster was 17.167 and it included 37 nodes and 309 edges (Fig. 3A). Furthermore, genes involved in this module were analyzed using the DAVID online platform for GO and KEGG analysis. The results indicated that genes in this module were significantly enriched in 'SLE', 'muscle filament sliding' and 'nucleosome’ (Fig. 3B, Table I). 

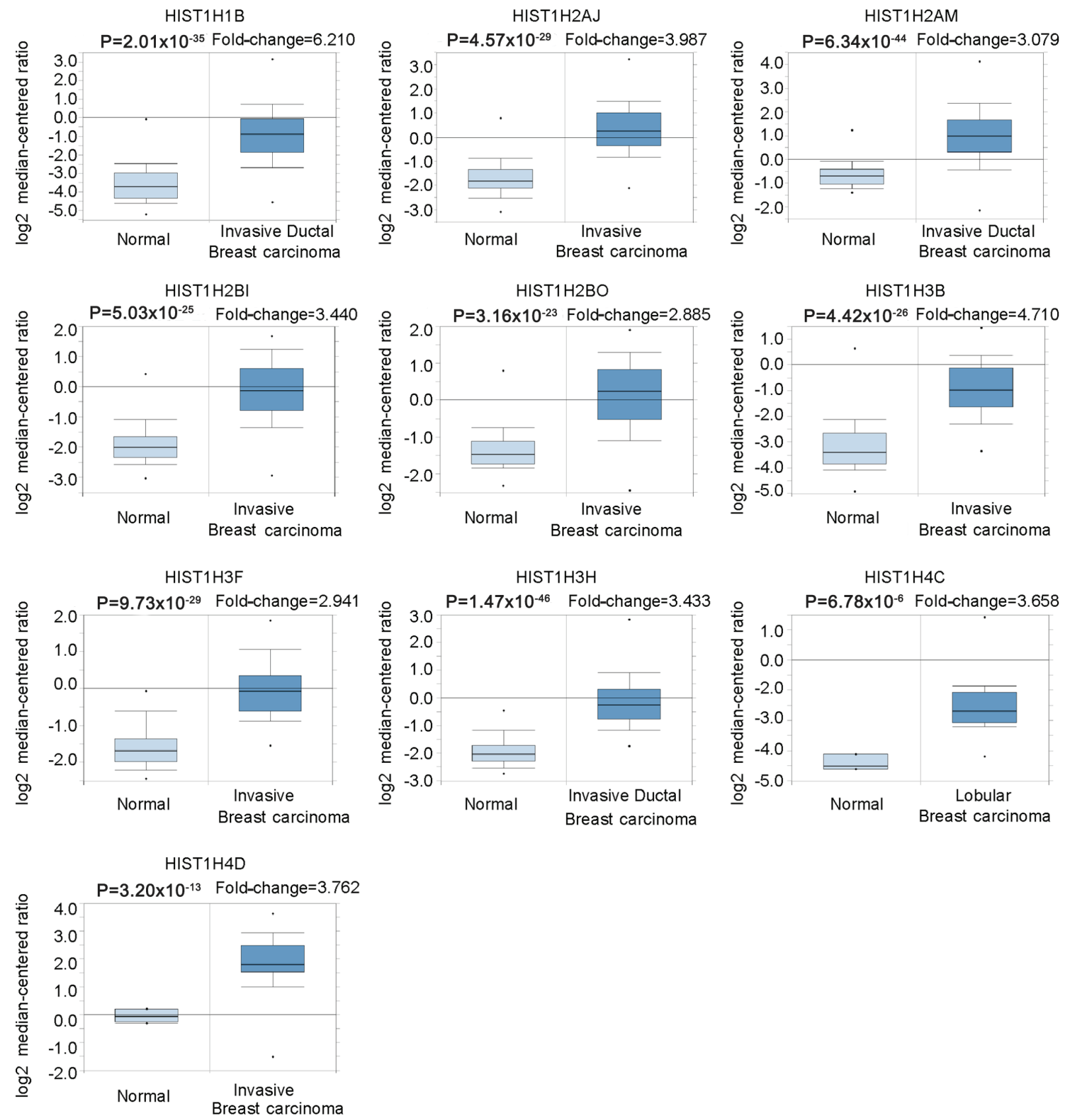

Figure 4. Expression levels of hub genes compared between different types of breast cancer and normal tissues from the Oncomine platform. Fold-changes and P-values of each hub gene are displayed in the plot.

From the MCODE plug-in, a total of 10 genes were selected as hub genes with degrees $\geq 10$. The further analysis focused on histone family genes, which were all upregulated in BC in the present results (Table SIII). The names of the hub genes were as follows: Histone cluster $1 \mathrm{H} 1$ family member B (HIST1H1B), HIST1H2AJ, HIST1H2AM, HIST1H2BI, HISTIH2BO, HISTIH3B, HISTIH3F, HISTIH3H, HISTIH4C and HISTIH4D.

Hub gene analysis. Oncomine was used to further verify the expression levels of 10 hub genes in $\mathrm{BC}$ vs. normal breast tissues. The results indicated that the histone family genes selected were significantly upregulated in invasive breast carcinoma, invasive ductal breast carcinoma and lobular breast carcinoma, with $\mathrm{P}<0.05$ considered to indicate statistical significance (Fig. 4; Table SIV). Analysis using the HPA database indicated that the 10 hub genes were slightly elevated in BC tissues (Fig. 5; Table III).

Prognostic value of histone family genes in $B C$. PrognoScan was used to further investigate the survival of hub genes in BC patients. The present results demonstrated that a higher expression of HIST1H2AJ (Cox $\mathrm{P}=0.002962)$, HISTIH2AM (Cox $\mathrm{P}=0.019590)$, HIST1H2BI (Cox $\mathrm{P}=0.019661)$, HIST1H2BO (Cox $\mathrm{P}=0.016204)$, HIST1H3H (Cox $\mathrm{P}=0.049099)$ and HISTIH4C (Cox $\mathrm{P}=0.012216)$ were associated with poorer overall survival for BC patients. Higher expression of HIST1H2BI (Cox $\mathrm{P}=0.043480)$, HISTIH2BO (Cox $\mathrm{P}=0.048887)$, HIST 1 H3H (Cox $\mathrm{P}=0.026703)$, HISTIH3F $(\operatorname{Cox} \mathrm{P}=0.024383)$ and 


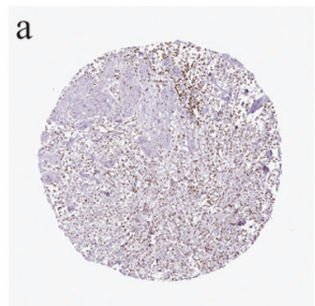

HIST1H1B

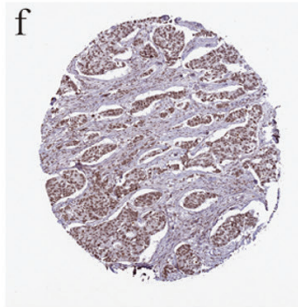

HIST1H3B

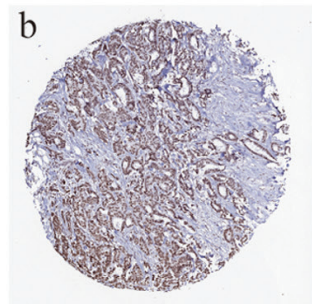

HIST1H2AJ

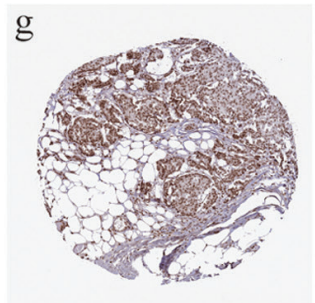

HIST1H3F

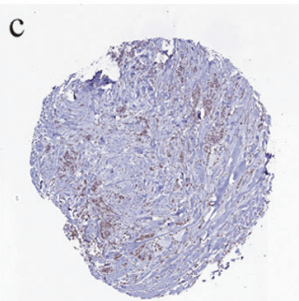

HIST1H2AM

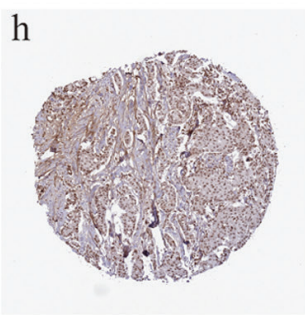

HIST1H3H

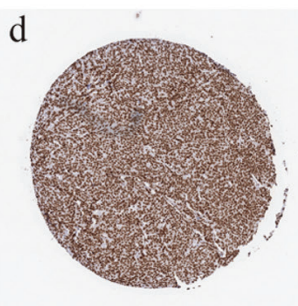

HIST1H2BI

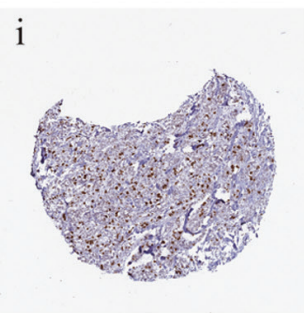

HIST1H4C

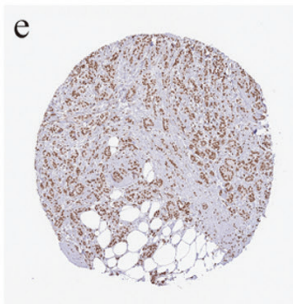

HIST1H2BO

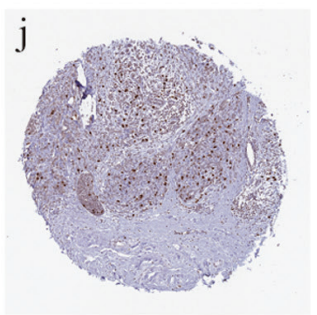

HIST1H4D

Figure 5. Immunohistochemical analysis of the histone family gene in breast cancer tissues from the Human Protein Atlas. (a) The protein expression of HIST1H1B. (b) The protein expression of HIST1H2AJ. (c) The protein expression of HIST1H2AM. (d) The protein expression of HIST1H2BI. (e) The protein expression of HIST1H2BO. (f) The protein expression of HIST1H3B. (g) The protein expression of HIST1H3F. (h) The protein expression of HIST1H3H. (i) The protein expression of HIST1H4C. (j) The protein expression of HIST1H4D. HIST1, histone cluster 1.

Table III. Immunohistochemistry analysis of histone family gene.

\begin{tabular}{|c|c|c|c|c|c|c|}
\hline Gene & Patient ID & Age & Sex & Cancer type & Intensity & Quantity \\
\hline HIST1H1B & 1910 & 61 & Female & Breast Duct carcinoma & Moderate & $>75 \%$ \\
\hline HIST1H2AJ & 1939 & 87 & Female & Breast Duct carcinoma & Strong & $>75 \%$ \\
\hline HIST1H2AM & 2091 & 40 & Female & Breast Duct carcinoma & Moderate & $>75 \%$ \\
\hline HIST1H2BI & 1775 & 55 & Female & Breast Duct carcinoma & Strong & $>75 \%$ \\
\hline HIST1H2BO & 2115 & 73 & Female & Breast Duct carcinoma & Moderate & $>75 \%$ \\
\hline HIST1H3B & 2160 & 83 & Female & Breast Duct carcinoma & Moderate & $>75 \%$ \\
\hline HIST1H3F & 2428 & 75 & Female & Breast Duct carcinoma & Strong & $>75 \%$ \\
\hline HIST1H3H & 1874 & 80 & Female & Breast Duct carcinoma & Strong & $>75 \%$ \\
\hline HIST1H4C & 2805 & 59 & Female & Breast Lobular carcinoma & Strong & $75-25 \%$ \\
\hline HIST1H4D & 3546 & 58 & Female & Breast Lobular carcinoma & Strong & $<25 \%$ \\
\hline
\end{tabular}

HIST1, histone cluster 1.

HIST1H4D (Cox $\mathrm{P}=0.031189)$ was associated with poorer relapse-free survival. Higher expression of HIST1H1B (Cox $\mathrm{P}=0.021894), H I S T 1 H 3 H(\mathrm{Cox} \mathrm{P}=0.02670), H I S T 1 H 3 F(\mathrm{Cox}$ $\mathrm{P}=0.000753), H I S T 1 H 3 B(\mathrm{Cox} \mathrm{P}=0.000020), H I S T 1 H 4 C$ (Cox $\mathrm{P}=0.000046)$, HIST1H4D (Cox $\mathrm{P}=0.031189)$ was associated with poorer distant metastasis-free survival (Fig. 6). Cox P-values and hazard ratios with $95 \%$ confidence intervals are displayed in Table SV.

Expression of hub genes in BC. Next, seven of the 10 hub genes were selected to analyze the expression levels in seven $\mathrm{BC}$ samples and 10 para-carcinoma tissues by qPCR. When compared with that in normal breast tissues, the levels of HIST1H1B, HIST1H2BI, HIST1H2BO and HIST1H3F were significantly increased in $\mathrm{BC}$ samples compared to paracarcinoma samples $(\mathrm{P}=0.0016, \mathrm{P}=0.0220, \mathrm{P}=0.0323$ and $\mathrm{P}=0.0184$, respectively; Fig. 7). However, the expression levels of HISTIH3B, HISTIH4C and HISTIH4D in these samples were not significantly different from those in the adjacent tissues (P>0.05; Fig. S3).

Relationship between genes and clinical pathological parameters. A total of 1,096 BC samples from TCGA were investigated to explore the relationship between gene expression and clinical pathological characteristics. As can be seen in Table SVI, a significant difference in HIST1H1B was due to age $(\mathrm{P}<0.001)$, estrogen responsive $(\mathrm{ER})$ growth status $(\mathrm{P}<0.001)$, progesterone responsive growth $(\mathrm{PR})$ status $(\mathrm{P}<0.001)$, human epidermal growth factor 2 (HER2) status $(\mathrm{P}=0.003)$ and primary tumors $(\mathrm{T})(\mathrm{P}=0.032)$. $\mathrm{BC}$ patients in the group aged $<60$ (median $=5.85)$ had an increased expression of HIST1H1B compared with those aged $\geq 60$ 

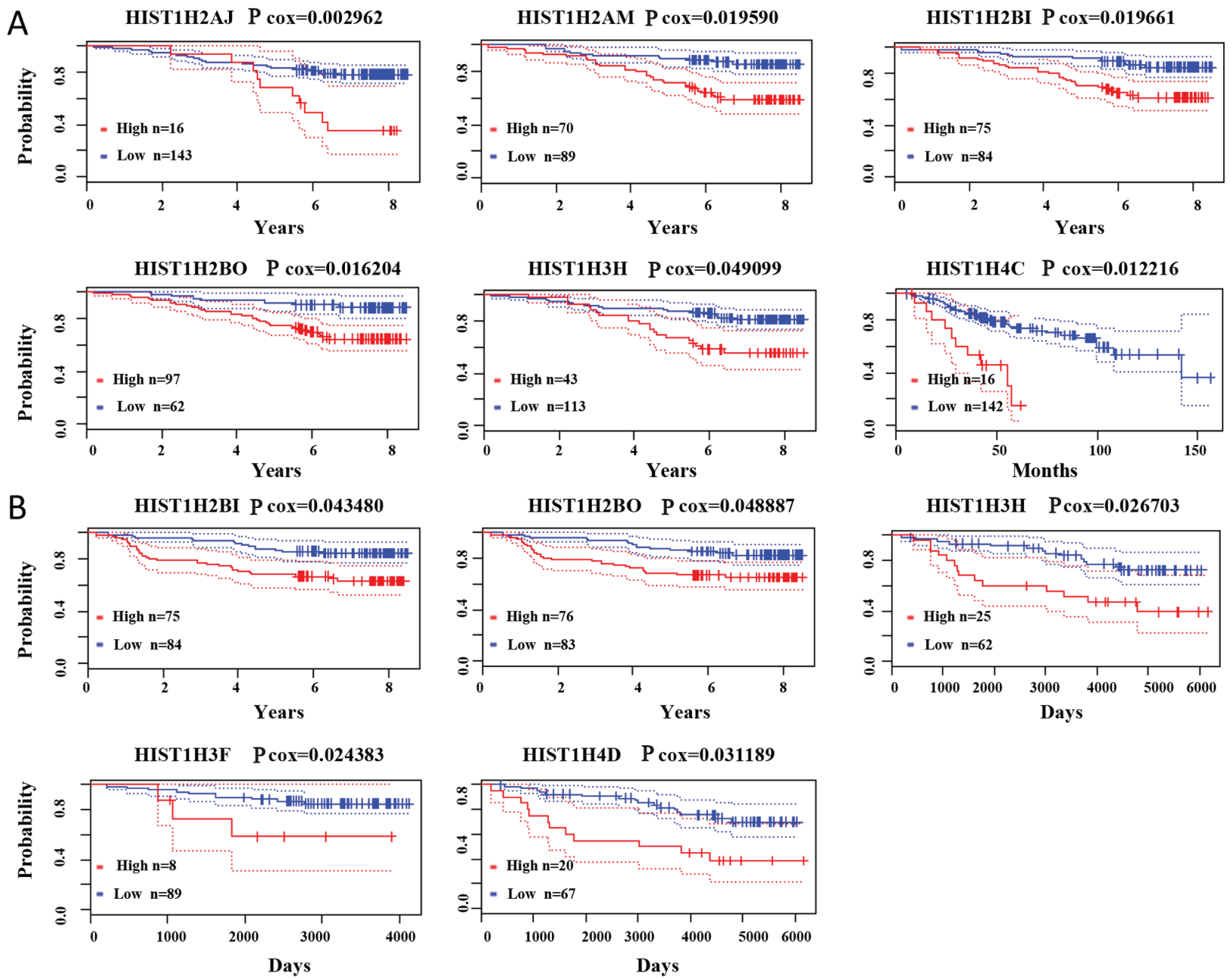

C

HIST1H1B P cox $=0.021894$

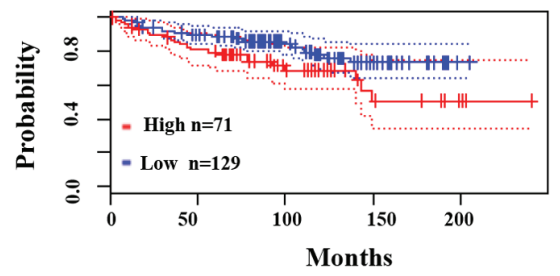

HIST1H3H $P \operatorname{cox}=0.026703$

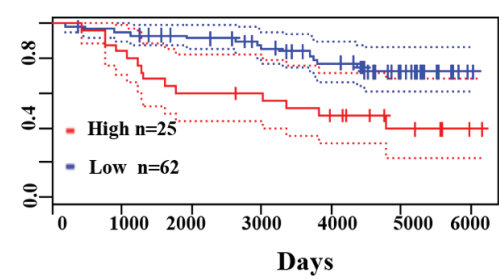

HIST1H3F P cox $=0.000753$

HIST1H3B $P \operatorname{cox}=0.000020$

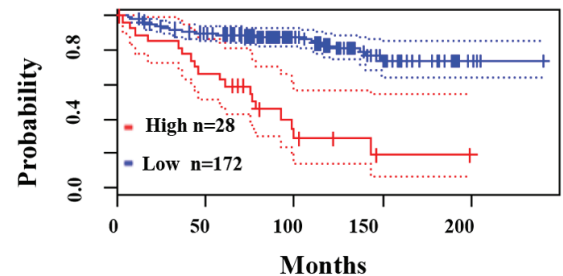

HIST1H4C P $\operatorname{cox}=0.000046$
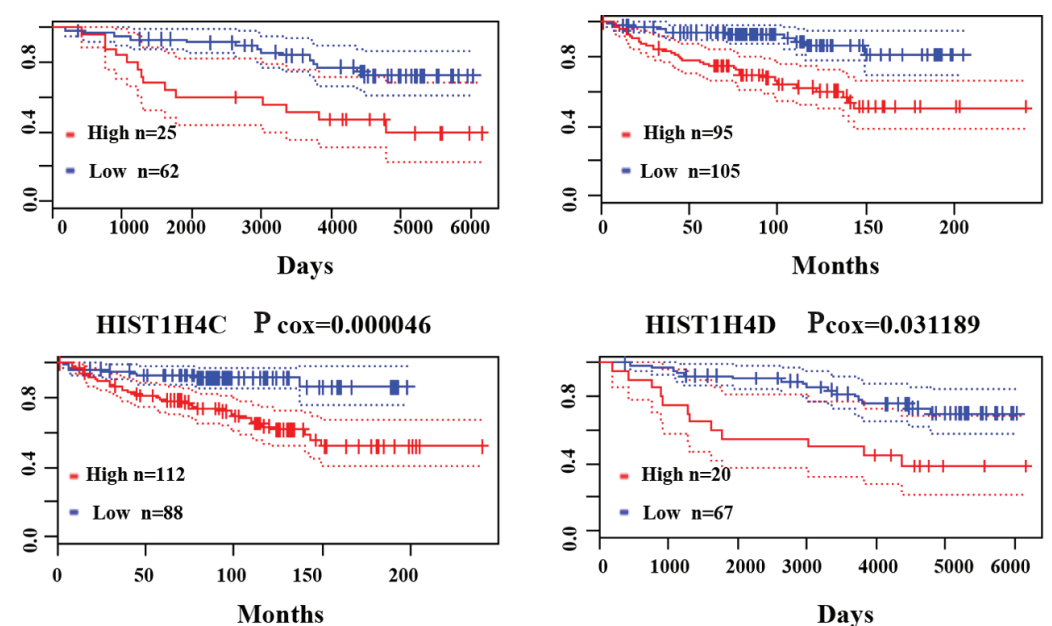

\section{HIST1H4D Pcox $=0.031189$}

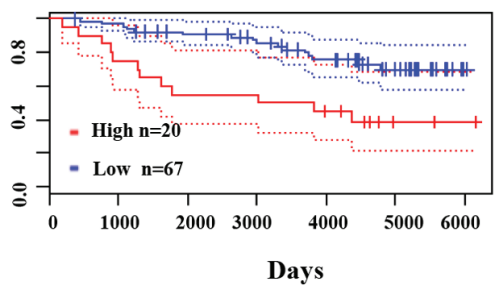

Figure 6. Association between histone family gene set expression and prognosis in breast cancer patients from the PrognoScan database. Kaplan-Meier curve analysis for (A) overall survival, (B) relapse-free survival and (C) distant metastasis-free survival. Cox P<0.05. HIST1, histone cluster 1.

(median=3.83). The expression of HIST1H1B in ER negative BC patients (median=7.99) was more significantly increased than that in ER positive patients (median=4.07). The expression of HIST1H1B in PR negative BC patients (median=7.32) was increased when compared with expression in PR positive patients (median=4.02). Patients at an early stage (T1-T2; median=5.15) had increased expression compared with those at an advanced stage (T3-T4; median=4.17). As can be seen in Table SVII, a significant difference in HIST1H2BI expression was due to ER Status ( $\mathrm{P}=0.047)$. ER positive $\mathrm{BC}$ patients (median $=0.95)$ had increased expression of HIST1H2BI compared with the ER negative group (median=0.82). Furthermore, as is shown in Table SVIII, a significant difference in HIST1H2BO was found to be related to age 


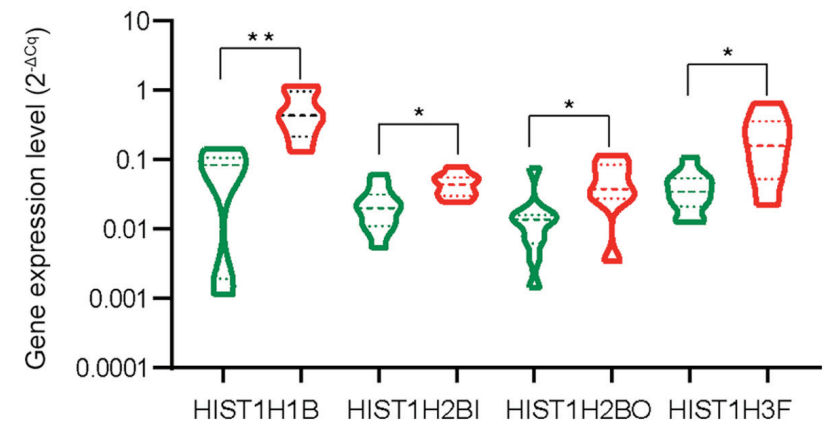

Figure 7. Violin plot of hub gene expression levels in breast cancer and normal tissues. The $\mathrm{x}$-axis represents hub genes and the $\mathrm{y}$-axis represents gene expression. ${ }^{*} \mathrm{P}<0.05$ and ${ }^{* *} \mathrm{P}<0.01$. HIST1, histone cluster 1.

$(\mathrm{P}<0.001)$, ER Status $(\mathrm{P}=0.001), \mathrm{PR}$ Status $(\mathrm{P}=0.003)$ and metastasis $(\mathrm{M})(\mathrm{P}=0.001)$. Patients aged $<60$ (median=15.95) exhibited increased expression of HIST1H2BO compared with patients aged $\geq 60$ (median=11.02). The expression of HIST1H2BO in ER negative BC patients (median=17.52) was more significantly increased than that in ER positive patients (median=12.91). The expression of HIST1H2BO in PR positive patients (median=18.09) was more significantly increased than in PR negative patients (median=13.94). The expression of HIST1H2BO in patients without metastasis (median=14.31) was more significantly increased than in patients with metastasis (median=8.88). However as is revealed in Table SIX, there was no significant difference in HIST1H3F for clinicopathological parameters.

\section{Discussion}

Breast carcinoma is the most common type of malignant tumor in women worldwide. It has been classified into multiple subtypes according to the molecular status and its incidence has increased in recent years (2). Gene mutations, which may be inherited, are thought to be the most common etiological factor for BC (27). However, epigenetic reprogramming, which includes DNA methylation, histone modifications and RNA-mediated gene silencing, has gained vast interest from researchers investigating its role in $\mathrm{BC}$ development, drug resistance and clinical prognosis (28). Histone modifications occurring on lysine residues include acetylation, methylation, phosphorylation, sumoylation, biotinylation and ubiquitination (29).

In the present study, data were extracted from TCGA and 366 upregulated DEGs and 155 downregulated DEGs between $\mathrm{BC}$ and normal tissue samples were identified using bioinformatics. The PPI network of these DEGs was constructed and MCODE was used to construct clusters, which are closely and highly connected regions. The cluster with the highest score was selected and 37 genes were contained in this cluster. These genes were obviously enriched in SLE. Therefore, histone family genes were determined as hub genes in BC. Previous studies revealed that histone family genes are involved in multiple cancer types. Copy number variations of HISTIHIB were reported to be associated with cellular development and growth, and with proliferation in melanoma (30). HIST1H3B, as an amplification-dependent driver oncogene, was reported to be overexpressed in liver cancer (31). HISTIH3F, as a clas- sifier gene, was indicated to be able to predict the prognosis of laryngeal cancer patients (32). Furthermore, the mutation of histone $\mathrm{H} 3$ variants may be a potential specific therapeutic target for diffuse intrinsic pontine glioma (33). Downregulation of histone $\mathrm{H} 2 \mathrm{~A}$ and $\mathrm{H} 2 \mathrm{~B}$ may be a possible means of reversing clinical anthracycline resistance in BC (34). In addition, histone modification profiling may provide valuable classification biomarkers and predict the risk of BC subtypes (35). Li et al (13) revealed that the histone family of genes may serve as prognostic factors for survival prediction in patients with cervical cancer. The authors of the present study searched PubMed and found that the use of TCGA data for histones gene family in $\mathrm{BC}$ has not been studied, which means data on histones has not been investigated before in flagship TCGA papers to the best of our knowledge. The present analysis indicated that histone family genes may also be used as prognostic factors for BC patients. It is suggested that the histone family of genes is closely associated with gynecological cancer types.

According to the KEGG functional pathway analysis, the set of upregulated histone variant genes were mainly enriched in the SLE pathway. Histone modification-mediated chromatin changes and gene expression have a vital role in the pathophysiology of SLE, which is a systemic autoimmune disease (36). Global histone $\mathrm{H} 3$ and $\mathrm{H} 4$ hypoacetylation were associated with active cluster of differentiation $4^{+} \mathrm{T}$ cells in SLE (37). Deoxyribose-modified H2A histone bound by serum anti-DNA autoantibodies may trigger immune responses in SLE (38). Of note, an international multicenter cohort study suggested a small increased risk for cancer in general in SLE; however, a decreased risk was estimated for breast, endometrial and ovarian cancers (39). However, the specific molecular biological mechanisms of the roles of SLE pathways in BC require further study.

In the present study, 7 clinical $\mathrm{BC}$ and 10 adjacent noncancerous tissues were used to examine the levels of histone members using qPCR. HIST1H1B, HIST1H2BI, HIST1H2BO and HISTIH3F expression in BC had a tendency to be upregulated, which was consistent with the results of the analysis of TCGA data. However, the small number of samples is a limitation. In further studies, larger cohorts of BC patients are required to demonstrate the prognostic value of the genes identified by analysis of in-house data.

To the best of our knowledge, the present study provides the first preliminary screening to indicate the predictive value of histone members regarding the prognosis of $\mathrm{BC}$ patients. Through retrieval and analysis of gene expression and survival data of multiple patients with $\mathrm{BC}$, the present study enhances the understanding of histone members and their predictive value in $\mathrm{BC}$ prognosis. The present study provides evidence that the histone gene set may act as prognostic factors for survival in BC patients.

However, correlations between the clinical features and the histone gene set of $\mathrm{BC}$ have been seldom reported. The present study used a larger scale sample from TCGA breast cancer for a systematic investigation of the relationships. Therefore, based on the TCGA data, the HISTIH1B, HIST1H2BI, HIST1H2BO expression level in $\mathrm{BC}$ was related to age, ER status, PR status, HER2 status, stage, $\mathrm{T}$ and $\mathrm{M}$.

In conclusion, the present study identified differentially expressed mRNAs in BC. Of note, histone family genes were 
identified as the hub genes, which may have a significant impact on the survival and prognosis of $\mathrm{BC}$ patients. However, the biological function of histone family genes in $\mathrm{BC}$ requires further research.

\section{Acknowledgements}

Not applicable.

\section{Funding}

The present study was supported by the Shanghai Talents Development Foundation (grant no. 2017103), the National Key R\&D Program of China (grant no. 2016YFC0104303) and the National Natural Science Fund (grant no. 81771859).

\section{Availability of data and materials}

All data generated or analyzed during this study are included in this published article or are available from the stated public repository.

\section{Authors' contributions}

FY and CM designed the study. WX, JZ and PZ wrote the manuscript and analyzed the data. SQ, HZ, XF and YY performed the experiments. RL, HL, YH, YL, XY and ZL collected patient samples. All authors agree with the results and conclusions of this manuscript.

\section{Ethics approval and consent to participate}

The acquisition of tissue specimens for the present study was approved by the Ethical Committee of Shanghai Tenth People's Hospital (approval no. 107 SHSY-IEC-4.0/19-24/01). Each patient provided written informed consent prior to participating in the study.

\section{Patient consent for publication}

Not applicable.

\section{Competing interests}

The authors declare that they have no competing interests.

\section{References}

1. Bray F, Ferlay J, Soerjomataram I, Siegel RL, Torre LA and Jemal A: Global cancer statistics 2018: GLOBOCAN estimates of incidence and mortality worldwide for 36 cancers in 185 countries. CA Cancer J Clin 68: 394-424, 2018.

2. DeSantis CE, Ma J, Goding Sauer A, Newman LA and Jemal A: Breast cancer statistics, 2017, racial disparity in mortality by state. CA Cancer J Clin 67: 439-448, 2017.

3. Reed E and Corner J: Defining the illness trajectory of metastatic breast cancer. BMJ Support Palliat Care 5: 358-365, 2015.

4. Redig AJ and McAllister SS: Breast cancer as a systemic disease: A view of metastasis. J Intern Med 274: 113-126, 2013.

5. McShane LM, Altman DG, Sauerbrei W, Taube SE, Gion M and Clark GM; Statistics Subcommittee of the NCI-EORTC Working Group on Cancer Diagnostics: REporting recommendations for tumour MARKer prognostic studies (REMARK). Br J Cancer 93: 387-391, 2005 .
6. Costa-Pinheiro P, Montezuma D, Henrique R and Jerónimo C: Diagnostic and prognostic epigenetic biomarkers in cancer. Epigenomics 7: 1003-1015, 2015.

7. Dworkin AM, Huang TH and Toland AE: Epigenetic alterations in the breast: Implications for breast cancer detection, prognosis and treatment. Semin Cancer Biol 19: 165-171, 2009.

8. Karsli-Ceppioglu S, Dagdemir A, Judes G, Ngollo M, PenaultLlorca F, Pajon A, Bignon YJ and Bernard-Gallon D: Epigenetic mechanisms of breast cancer: An update of the current knowledge. Epigenomics 6: 651-664, 2014.

9. Yang HD, Kim PJ, Eun JW, Shen Q, Kim HS, Shin WC, Ahn YM, Park WS, Lee JY and Nam SW: Oncogenic potential of histone-variant H2A.Z.1 and its regulatory role in cell cycle and epithelial-mesenchymal transition in liver cancer. Oncotarget 7: 11412-11423, 2016.

10. Rangasamy D: Histone variant H2A.Z can serve as a new target for breast cancer therapy. Curr Med Chem 17: 3155-3161, 2010.

11. Chen H, Lorton B, Gupta V and Shechter D: A TGF $\beta$ PRMT5-MEP50 axis regulates cancer cell invasion through histone $\mathrm{H} 3$ and $\mathrm{H} 4$ arginine methylation coupled transcriptional activation and repression. Oncogene 36: 373-386, 2017.

12. Yokoyama Y, Matsumoto A, Hieda M, Shinchi Y, Ogihara E, Hamada $M$, Nishioka $Y$, Kimura $H$, Yoshidome $K$, Tsujimoto M, et al: Loss of histone H4K20 trimethylation predicts poor prognosis in breast cancer and is associated with invasive activity. Breast Cancer Res 16: R66, 2014.

13. Li X, Tian R, Gao H, Yang Y, Williams BRG, Gantier MP, McMillan NAJ, Xu D, Hu Y and Gao Y: Identification of a histone family gene signature for predicting the prognosis of cervical cancer patients. Sci Rep 7: 16495, 2017.

14. Tomczak K, Czerwińska P and Wiznerowicz M: The Cancer Genome Atlas (TCGA): An immeasurable source of knowledge. Contemp Oncol (Pozn) 19: A68-A77, 2015.

15. Robinson MD, McCarthy DJ and Smyth GK: edgeR: A Bioconductor package for differential expression analysis of digital gene expression data. Bioinformatics 26: 139-140, 2010.

16. Huang W, Sherman BT and Lempicki RA: Bioinformatics enrichment tools: Paths toward the comprehensive functional analysis of large gene lists. Nucleic Acids Res 37: 1-13, 2009.

17. Huang W, Sherman BT and Lempicki RA: Systematic and integrative analysis of large gene lists using DAVID bioinformatics resources. Nat Protoc 4: 44-57, 2009.

18. Ashburner M, Ball CA, Blake JA, Botstein D, Butler H, Cherry JM, Davis AP, Dolinski K, Dwight SS, Eppig JT, et al; The Gene Ontology Consortium: Gene ontology: Tool for the unification of biology. Nat Genet 25: 25-29, 2000.

19. Gene Ontology Consortium: Gene Ontology Consortium: Going forward. Nucleic Acids Res 43: D1049-D1056, 2015.

20. Kanehisa M, Furumichi M, Tanabe M, Sato Y and Morishima K: KEGG: New perspectives on genomes, pathways, diseases and drugs. Nucleic Acids Res 45: D353-D361, 2017.

21. Szklarczyk D, Morris JH, Cook H, Kuhn M, Wyder S, Simonovic M, Santos A, Doncheva NT, Roth A, Bork P, et al: The STRING database in 2017: Quality-controlled protein-protein association networks, made broadly accessible. Nucleic Acids Res 45: D362-D368, 2017.

22. Van Parys T, Melckenbeeck I, Houbraken M, Audenaert P, Colle D, Pickavet M, Demeester P and Van de Peer Y: A Cytoscape app for motif enumeration with ISMAGS. Bioinformatics 33: 461-463, 2017.

23. Rhodes DR, Kalyana-Sundaram S, Mahavisno V, Varambally R, Yu J, Briggs BB, Barrette TR, Anstet MJ, Kincead-Beal C, Kulkarni P, et al: Oncomine 3.0: Genes, pathways, and networks in a collection of 18,000 cancer gene expression profiles. Neoplasia 9: 166-180, 2007.

24. Pontén F, Jirström K and Uhlen M: The Human Protein Atlas - a tool for pathology. J Pathol 216: 387-393, 2008.

25. Mizuno H, Kitada K, Nakai K and Sarai A: PrognoScan: A new database for meta-analysis of the prognostic value of genes. BMC Med Genomics 2: 18, 2009.

26. Tang R, Liang L, Luo D, Feng Z, Huang Q, He R, Gan T, Yang L and Chen G: Downregulation of miR-30a is associated with poor prognosis in lung cancer. Med Sci Monit 21: 2514-2520, 2015.

27. Abbasi S, Rasouli M, Nouri M and Kalbasi S: Association of estrogen receptor- $\alpha$ A908G (K303R) mutation with breast cancer risk. Int J Clin Exp Med 6: 39-49, 2013.

28. Basse $\mathrm{C}$ and Arock $\mathrm{M}$ : The increasing roles of epigenetics in breast cancer: Implications for pathogenicity, biomarkers, prevention and treatment. Int J Cancer 137: 2785-2794, 2015. 
29. Katz TA, Huang Y, Davidson NE and Jankowitz RC: Epigenetic reprogramming in breast cancer: From new targets to new therapies. Ann Med 46: 397-408, 2014.

30. Fidalgo F, Rodrigues TC, Silva AG, Facure L, de Sá BC, Duprat JP, Achatz MI, Rosenberg C, Carraro DM and Krepischi AC: Role of rare germline copy number variation in melanoma-prone patients. Future Oncol 12: 1345-1357, 2016.

31. Ohshima K, Hatakeyama K, Nagashima T, Watanabe Y, Kanto K, Doi Y, Ide T, Shimoda Y, Tanabe T, Ohnami S, et al: Integrated analysis of gene expression and copy number identified potential cancer driver genes with amplification-dependent overexpression in 1,454 solid tumors. Sci Rep 7: 641, 2017.

32. Mirisola V, Mora R, Esposito AI, Guastini L, Tabacchiera F, Paleari L, Amaro A, Angelini G, Dellepiane M, Pfeffer U, et al: A prognostic multigene classifier for squamous cell carcinomas of the larynx. Cancer Lett 307: 37-46, 2011.

33. Castel D, Philippe C, Calmon R, Le Dret L, Truffaux N, Boddaert N, Pagès M, Taylor KR, Saulnier $\mathrm{P}$, Lacroix L, et al: Histone H3F3A and HIST1H3B K27M mutations define two subgroups of diffuse intrinsic pontine gliomas with different prognosis and phenotypes. Acta Neuropathol 130: 815-827, 2015.

34. Braunstein M, Liao L, Lyttle N, Lobo N, Taylor KJ, Krzyzanowski PM, Kalatskaya I, Yao CQ, Stein LD, Boutros PC, et al: Downregulation of histone H2A and H2B pathways is associated with anthracycline sensitivity in breast cancer. Breast Cancer Res 18: 16, 2016.
35. Chen X, Hu H, He L, Yu X, Liu X, Zhong R and Shu M: A novel subtype classification and risk of breast cancer by histone modification profiling. Breast Cancer Res Treat 157: 267-279, 2016.

36. Hedrich CM: Epigenetics in SLE. Curr Rheumatol Rep 19: 58, 2017.

37. Hu N, Qiu X, Luo Y, Yuan J, Li Y, Lei W, Zhang G, Zhou Y, Su Y and Lu Q: Abnormal histone modification patterns in lupus CD $4^{+}$ T cells. J Rheumatol 35: 804-810, 2008

38. Alam S, Arif Z and Alam K: Glycated-H2A histone is better bound by serum anti-DNA autoantibodies in SLE patients: Glycated-histones as likely trigger for SLE? Autoimmunity 48: 19-28, 2015.

39. Bernatsky S, Ramsey-Goldman R, Labrecque J, Joseph L, Boivin JF, Petri M, Zoma A, Manzi S, Urowitz MB, Gladman D, et al: Cancer risk in systemic lupus: An updated international multi-centre cohort study. J Autoimmun 42: 130-135, 2013.

(c) (i) () This work is licensed under a Creative Commons Attribution-NonCommercial-NoDerivatives 4.0 International (CC BY-NC-ND 4.0) License. 